\title{
The Ameliorating Activity of Portulaca oleracea L. Polysaccharide on the UC Mouse Model Induced by DSS

\author{
Di Gao ${ }^{1}$, Yang Yang ${ }^{1}$, Rui Zhao ${ }^{1 *}$, Junyang $\mathrm{Cao}^{1}$, Gang $\mathrm{Ma}^{1}$, Chengbao $\mathrm{Pi}^{1}$, Zhen Jiang ${ }^{1}$
}

\begin{abstract}
${ }^{1}$ Department of Pharmaceutical Engineering, College of Life Science \& Biotechnology, Heilongjiang August First Land Reclamation University, Daqing High-Tech Industrial Development Zone, 163319, P. R. China
\end{abstract}

DOI: $10.36347 /$ sasjm.2021.v07i04.004

| Received: 14.03.2021 | Accepted: 21.04.2021 | Published: 27.04.2021

*Corresponding author: Rui Zhao

\section{Abstract}

\section{Original Research Article}

Ulcerative colitis (UC) is the chronic non-specific intestinal inflammatory disease. The purpose of this study is to establish a UC mouse model and explore the improvement effect of Portulaca oleracea L. polysaccharide (POL-P) on the UC experimental model. Dextran Sulphate Sodium Salt (DSS) concentration screening for establishing UC mouse model was used 2\% DSS, 3\% DSS and 4\% DSS, respectively. Disease activity index (DAI) was recorded daily. Mice were sacrificed at the end of the experiment. The length of the colon and morphological structure of colonic tissue were analyzed. To further evaluate the safety of POL-P, the morphology analysis of liver and kidney tissue sections was performed. The results showed that 4\% DSS was the optimal modeling concentration. POL-P had obvious prevention and treatment effects on UC mice. And POL-P has no toxic side effects on the liver and kidney tissues of mouse. The experimental results provide a theoretical basis for developing POL-P into a new drug with high efficiency and low toxicity for the prevention and treatment of UC.

Keywords: Ulcerative colitis; Portulaca oleracea L. polysaccharide; Dextran Sulphate Sodium Salt; Disease activity index

Copyright $\left({ }_{0} 2021\right.$ The Author(s): This is an open-access article distributed under the terms of the Creative Commons Attribution 4.0 International License (CC BY-NC 4.0) which permits unrestricted use, distribution, and reproduction in any medium for non-commercial use provided the original author and source are credited.

\section{INTRODUCTION}

Ulcerative colitis (UC) is the chronic non-specific intestinal inflammatory disease [1]. The incidence has increased dramatically with the acceleration of life rhythm, the change of diet structure and the improvement of detection level. Moreover, it is not easy to heal, leading to a high incidence of colon cancer [2, 3]. The treatment of UC with western medicine mainly focuses on immunosuppression and anti-inflammation. However, it will cause more adverse reactions because western medicine is used long-term repeated [4]. Portulaca oleracea L. is a kind of food and medicine homologous plant with the functions of clearing away heat and detoxification [5]. Clinical studies have found that Portulaca oleracea L. has significant efficacy in the treatment of UC, however, its reasonable and safe use is hindered by its unclear scientific connotation [6]. The purpose of this study is to establish a UC mouse model and explore the improvement effect of Portulaca oleracea L. polysaccharide (POL-P) on the UC mouse model.

\section{MATERIALS AND METHODS MATERIALS}

POL-P was obtained from Portulaca oleracea L. in our laboratory. The more details for preparation of water-soluble polysaccharide including separation, purification and identification refered to our previous work. Dextran Sulphate Sodium Salt (DSS) was obtained from MP Biomedical (Solon, OH, United States). All other reagents used were of analytical grade.

$\mathrm{BALB} / \mathrm{c}$ female mice (6-8 weeks) were provided by the Experimental Animal Center of Changchun. The animals were treated according to the National Institute of Health Guide for the Care and Use of Laboratory Animals. The study was approved by the Institutional Animal Care and Use Committee of Heilongjiang August First Land Reclamation University.

\section{DSS concentration screening for establishing UC mouse model}

Forty mice were divided randomly into 4 groups, including control group, 2\% DSS group, 3\% 
Di Gao et al., SAS J Med, Apr, 2021; 7(4): 139-144

DSS group, 4\% DSS group, ten mice in each group. Freely drink purified water for three days. On the 4th day, the mice in the control group drink purified water freely, and the mice in the model group drink freely drinking water containing $2 \%, 3 \%$, and $4 \%$ DSS. The mental signs and activity status in each group of mice were recorded every day and scored according to the table below. The final DAI score $=($ weight loss score + stool trait score + stool blood condition score) / 3 .

Table-1: DAI Scoring Criteria

\begin{tabular}{|l|l|l|l|}
\hline Scoring & Falling in body mass \% & Stool status & Blood in the stool \\
\hline 0 & 0 & normal & negative $(-)$ \\
\hline 1 & $0 \sim 5$ & fall in between & Fall in between \\
\hline 2 & $5 \sim 10$ & semi-sparse & occult blood $(+)$ \\
\hline 3 & $10 \sim 15$ & fall in between & fall in between \\
\hline 4 & $\geq 15$ & loose stool & gross bloody stool \\
\hline
\end{tabular}

\section{HE staining}

Mice were sacrificed and the liver, kidney, colon tissues were collected. The intestinal contents were rinsed with PBS solution. After rinsing, both ends of the colon tissue were repaired with a blade to remove the rolled and damaged parts during rinsing, and fixed with neutral formalin solution for $24 \mathrm{~h}$. The paraffin sections were prepared by the following procedures, such as fixation, trimming, dehydration, transparency, paraffin impregnation, embedding and sectioning, and then HE staining was performed. The tissue changes of liver, kidney and colon were observed under a microscope.

\section{Preventive and therapeutic effects of POL-P on UC mice}

Sixty mice were fed normally for one week and randomly divided into 6 groups with 10 mice in each group. The positive antimicrobial peptide group was given with $0.2 \mathrm{~g} / \mathrm{mL}, 0.2 \mathrm{~mL} / 10 \mathrm{~g} /$ time, once a day by intragastric administration. POL-P low-dose group $(0.2 \mathrm{~g} / \mathrm{mL})$, POL-P medium-dose group $(0.4 \mathrm{~g} / \mathrm{mL})$ and POL-P high-dose group $(0.8 \mathrm{~g} / \mathrm{mL})$ were given by intragastric administration at $0.2 \mathrm{~mL} / 10 \mathrm{~g} /$ time, once a day, respectively. The experiment lasted for 12 days. On the first 5 days of the experiment, mice in the control group and model group were given normal saline by intragastric administration while drinking normal water, and the positive group and POL-P lowdose, medium-dose and high-dose groups were given intragastric administration at the above dose. On the 6th day of the experiment, except the control group, the UC mice model was established with $4 \%$ DSS water solution instead of drinking water in other groups, and continued to be gavage until the last day of the experiment according to the method of the previous 5 days. Mental signs and activity status of mice in each group were recorded every day and scored according to the following table, and the final DAI score = (body weight loss score + fecal traits score + stool blood condition score) $/ 3$.

\section{RESULT}

\section{DAI score results}

The results of DAI score were showed in Figure-1. The fecal particles were clear and the degree of soft and hard was suitable in the control group (Figure 1A), while the feces was soft and severe hematochezia and even serious anal bleeding were observed in the 4\% DSS group (Figure-1B). DAI score results showed that the control group had a lower score, while $4 \%$ DSS group had a higher score. DAI score results showed that it was a higher score in $4 \%$ DSS group, compared with control group (Figure 1C, $\mathrm{P}<0.0001)$.

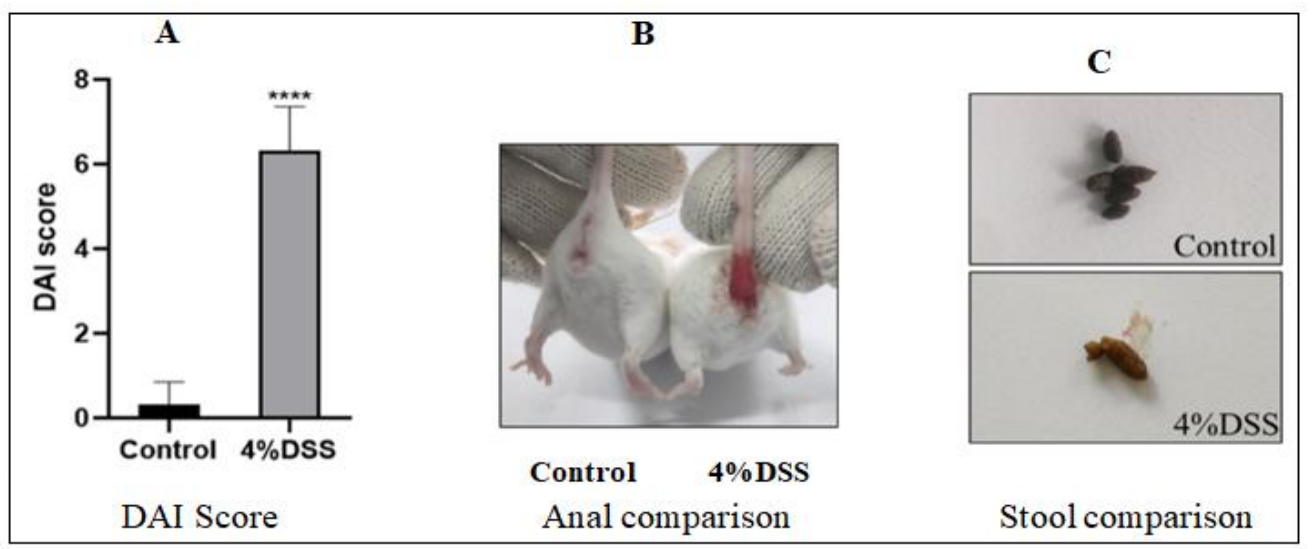

Fig-1: Comparison between control group and 4\% DSS group

Note: Compared with Control, ${ }^{\star \star \star \star} \mathrm{P}<0.0001$ 


\section{Colon changes in mice}

After the animals were sacrificed, the abdominal cavity was opened by cross method, and the whole intestine was carefully dissected for length measurement. The results showed in Figure-2, as a result of intestinal mucosal injury resulting in mucosal congestion and edema, the intestinal wall was thickened and the length of the intestine was shortened in the $4 \%$ DSS group. It is significantly different from the control group.

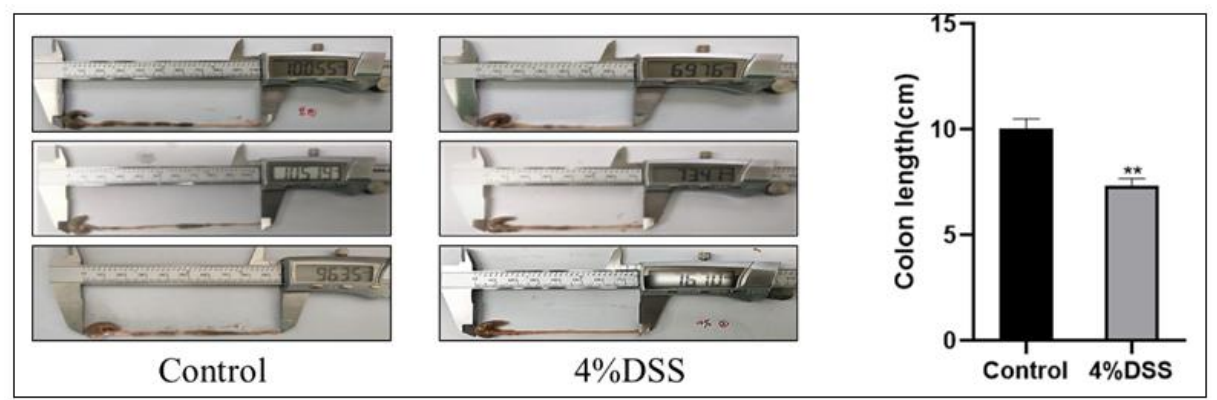

Fig-2: Colon length comparison between control group and 4\% DSS group

Note: Compared with Control, $\star \mathrm{P}<0.01$

\section{Colonic histopathological observation}

The results of colonic histopathology were showed in Figure 3. There was no obvious damage of colon tissue in the control group. In the $4 \%$ DSS treatment group, the number of goblet cells was decreased, and neutrophils had infiltrated the intestinal lamina propria. The presence of transmural inflammation and ulceration in the intestine indicated that the integrity of the intestinal epithelium had been severely compromised. Further, the colonic lesion has reached the submucosa with crypt glandolysis. Histopathological scores showed that there was a significant difference between the $4 \%$ DSS group and the control group.

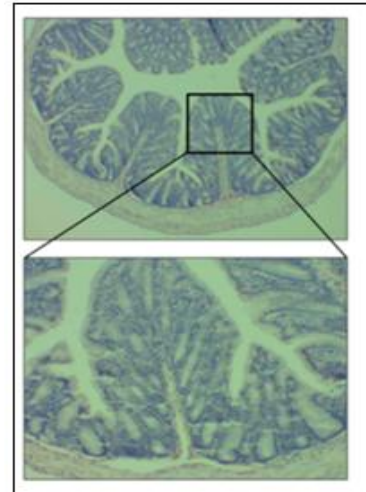

Control

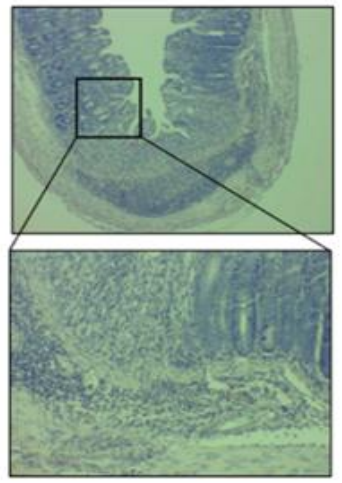

$4 \% \mathrm{DSS}$

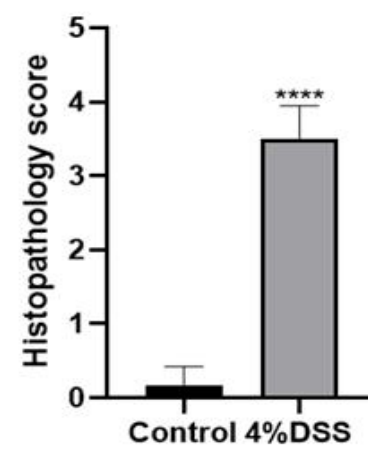

Fig-3: HE staining results and histopathological score

Note: Compared with Control, ${ }^{\star \star \star \star} \mathrm{P}<0.0001$

\section{The effect of POL-P on DAI score of UC mice}

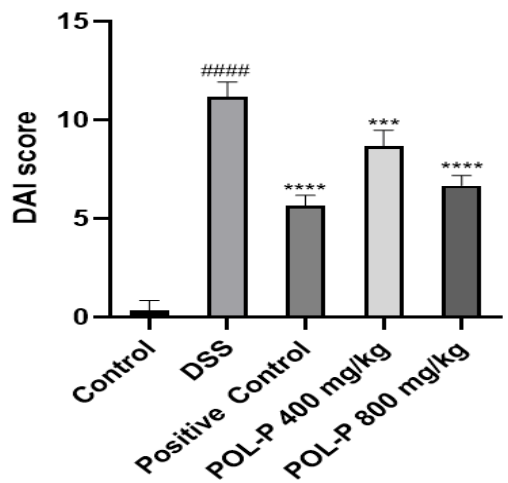

Fig-4: The effect of POL-P on the DAI score of the UC mouse model
Note: compared with Control, ${ }^{\# \# \# ~} \mathrm{P}<0.0001$; compared with DSS, ${ }^{\star \star \star}{ }^{\star} \mathrm{P}<0.05,{ }^{\star \star \star}{ }^{\star} \mathrm{P}<0.01$; compared with POL-P $800 \mathrm{mg}$ and $400 \mathrm{mg},{ }^{\star \star \star \star} \mathrm{P}<0.01$

The three basic indexes of weight loss, fecal viscosity and fecal occult blood were combined to evaluate the DAI score of mice. As showed in Figure-4, after DSS intervention, DAI index of model group, positive group and low, medium and high dose groups were significantly increased. The DAI score in the model group was the highest compared with other group mice. And the DAI index showed a downward trend with the increase of POL-P concentration. It suggested that POL-P had a certain therapeutic effect on UC mice, and the high-dose performed the best effect. 


\section{Effect of POL-P on colon length in UC mice}

The effect of POL-P on colon length in UC mice was showed in figure 5 . The average length of the colon in mouse of the model group was the shortest, and the length was elevated with the increase of POL-P concentration.

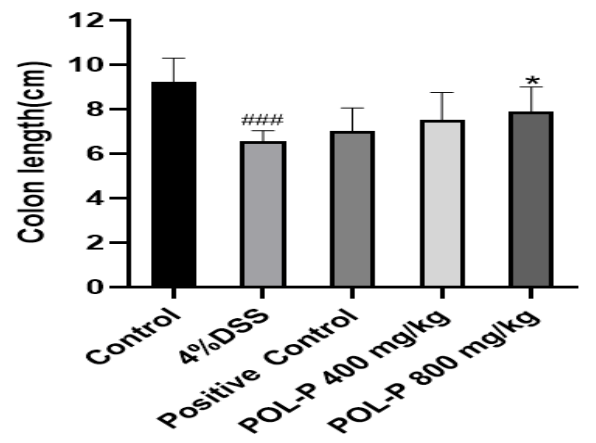

Fig-5: Effect of POL-P on colon length in UC mice Note: Compare with Control, ${ }^{\#} \mathrm{P}<0.05$; compare with $\mathrm{DSS},{ }^{\star} \mathrm{P}<0.05,{ }^{\star} \mathrm{P}<0.01,{ }^{\star \star}{ }^{\star} \mathrm{P}<0.001$
Effect of POL-P on colonic tissue of UC mice

The effects of POL-P on the morphological structure of colonic tissue were showed in Figure-6. The colonic mucosal epithelium was intact, and the glands in the lamina propria were normal in control group. In addition, there were many goblet cells in the mucosal epithelium, and no degenerative necrosis, no edema in the submucosa, and no infiltration of inflammatory cells were observed in control group. The obvious symptoms of ulcerative colitis in intestinal tract were appeared, such as thicken intestinal wall, denatured and necrotic epithelial cells, disappeared crypt, missing goblet cells, and numerous inflammatory cell infiltration in $4 \%$ DSS treatment group. The above pathological features were improved by treatment with POL-P. The pathological score in the model group was significantly higher than that of the control group. Compared with model group, pathological scores in POL-P groups were significantly decreased. These results suggested that POL-P could improve the intestinal mucosal injury induced by DSS.
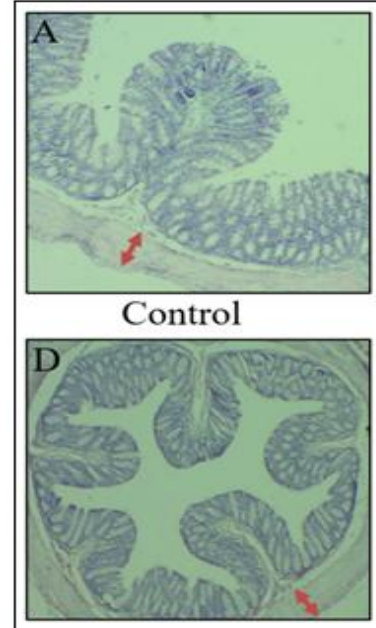

POL-P $400 \mathrm{mg} / \mathrm{kg}$

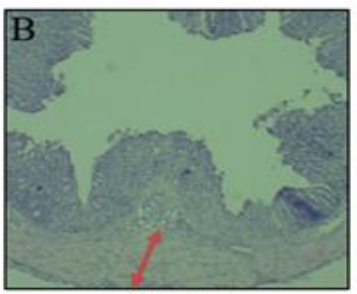

$4 \%$ DSS

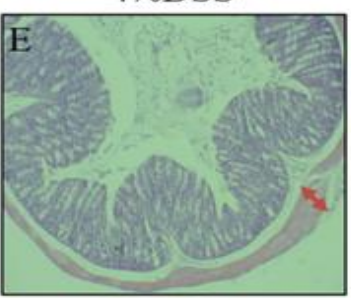

POL-P $800 \mathrm{mg} / \mathrm{kg}$

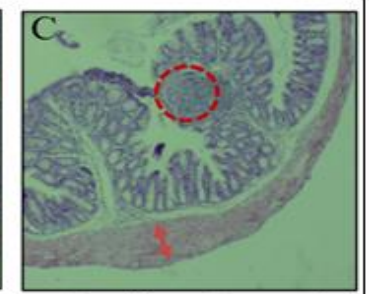

Positive Control

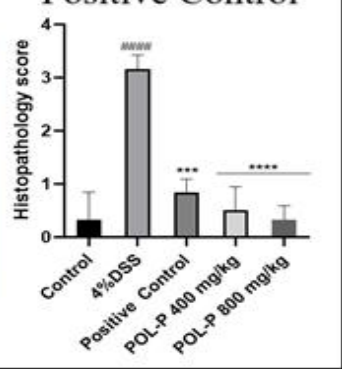

Fig-6: Effects of POL-P on colonic structure of UC mice

Note: Compare with Control, ${ }^{\# \# \# \#} \mathrm{P}<0.0001$; compare with DSS, ${ }^{\star \star} \mathrm{P}<0.001,{ }^{\star \star \star}{ }^{\star} \mathrm{P}<0.0001$

\section{Effect of POL-P on liver and kidney tissue of mice}

To further evaluate the safety of POL-P, the morphology analysis of liver and kidney tissue sections was performed. As showed in Figure-7, compared with the control group, the hepatic structure was complete without damage and central vein was in the center. The hepatic cords were arranged radially, and the liver cells were arranged neatly with normal volume, without inflammatory cell infiltration and necrotic cell generation. In addition, the renal tissue structure was normal, and the glomerular and tubular structures were clear and intact (Figure-8). The observation results suggested that POL-P had no obvious toxic effect on liver and kidney of mice.

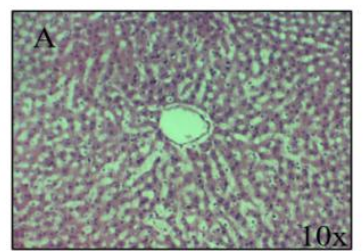

Control

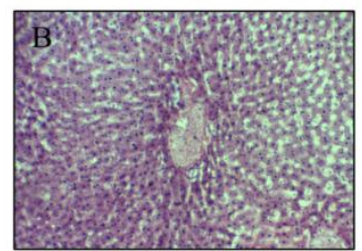

POL-P 400mg

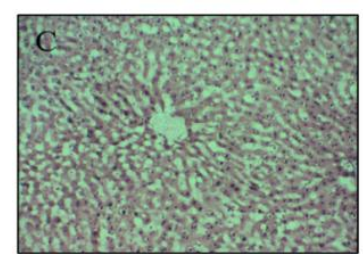

POL-P $800 \mathrm{mg}$

Fig-7: Effect of POL-P on liver tissue of mice $(20 \times 10)$ 


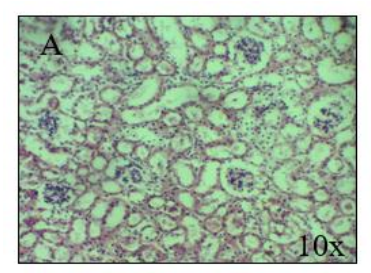

Control

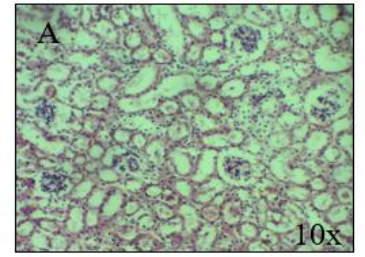

Control

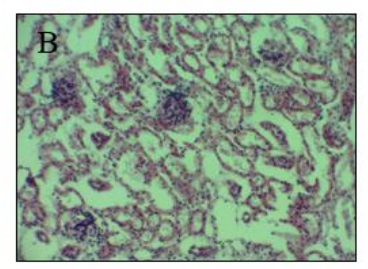

POL-P 400mg

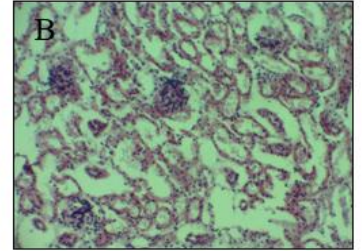

POL-P 400mg

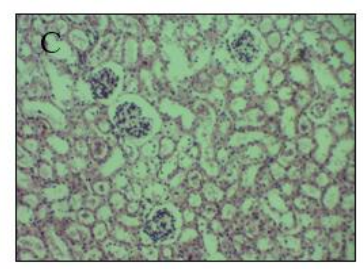

POL-P 800mg

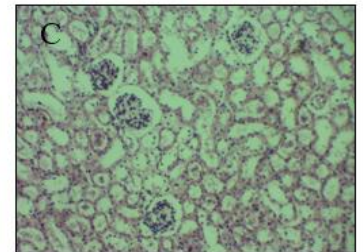

POL-P 800mg

Fig-8: Effect of POL-P on kidney tissue of mice $(20 \times 10)$

\section{DISCUSSION}

Ulcerative colitis is a chronic inflammatory bowel disease caused by multiple factors. It is characterized by high incidence, long course and repeated attacks. Its clinical manifestations are mainly abdominal pain, diarrhea and mucinous pus and bleeding stools [7]. UC tends to occur in the sigmoid colon and rectum, but serious cases can also involve the whole intestine, which seriously affects People's Daily life ${ }^{[9]}$. Studies have found that patients with inflammatory bowel disease (IBD), which has a disease duration of more than 30 months, has a cancer rate of $45 \%$ [8]. The epidemiological data at domestic and overseas show that the prevalence and incidence of UC is an obvious upward trend, and it has been listed as one of the modern refractory diseases by the World Health Organization [9]. In recent years, the incidence of UC in China has been rising and gradually getting younger. At present, the clinical treatment of UC in western medicine is mainly to control acute attacks, relieve colonic inflammation, and thus relieve symptoms of diarrhea, rectal bleeding and abdominal pain. However, these drugs are expensive and have serious side effects. It can not meet the needs of clinical application because it is prone to relapse after drug withdrawal and the treatment effect is not ideal for some refractory cases [10]. Therefore, it is urgent to find a kind of therapeutic drugs with definite curative effect, small toxic and side effects and long-term use.

In recent years, traditional Chinese medicine has a remarkable effect on the treatment of this disease. At the same time, it have many advantages such as low relapse rate, little adverse reaction, reduction of hormone dependence, and short course of treatment which has become a research hotspot. Portulaca oleracea is the whole grass of Portulaca oleracea L., which has the effects of heat-clearing and detoxifying [11]. Portulaca oleracea is rich in nutrition and is one of the wild medicinal materials with the same origin of medicine and food as recognized by the Ministry of Health of China [12, 13]. As a traditional gastrointestinal drug, it has been proved to be effective in the treatment of UC by years of clinical application $[14,15]$. However, studies on Portulaca oleracea in the treatment of UC mostly focus on extractum, water decoction and crude extract, and the effect of active ingredient on UC and its mechanism of action have not been reported yet. In this study, sodium dextran sulfate was used to establish a UC mice model. Polysaccharide, the active ingredient of Portulaca oleracea was prepared in our laboratory and was used to treat UC model mice with different doses. The improvement effect of POL-P on UC mice was determined by analyzing DAI, pathological damage of colon, inflammatory cell infiltration degree, villus length and the depth of crypt in each group. The results of this study provide a theoretical basis for revealing the clinical application of POL-P in the treatment of UC.

\section{CONCLUSION}

POL-P, the active ingredient of Portulaca oleracea L. had obvious prevention and treatment effects on UC mice induced by DSS. And POL-P has no toxic side effects on the liver and kidney tissues of mouse. The experimental results provide a theoretical basis for developing POL-P into a new drug with high efficiency and low toxicity for the prevention and treatment of UC.

\section{ACKNOWLEDGMENT}

The present study was funded by the Innovation and Entrepreneurship Training Program for College Students of Heilongjiang Province of China under Grant 202010223018; the Chinese National Natural Science Foundation Grant under Grant 31772789 .

\section{REFERENCES}

1. Ordás I, Eckmann L, Talamini M, Baumgart DC, Sandborn WJ. Ulcerative colitis. Lancet. 2012, 380(9853):1606-19.

2. Kaplan GG. The global burden of IBD: from 2015 
to 2025. Nat Rev Gastroenterol Hepatol, 2015, 12(12): 720-727.

3. Magro F, Gionchetti P, Eliakim R, Ardizzone S, Armuzzi A, Barreiro-de Acosta M, Burisch J, Gecse KB, Hart AL, Hindryckx P, Langner C. Third European evidence-based consensus on diagnosis and management of ulcerative colitis. Part 1: definitions, diagnosis, extra-intestinal manifestations, pregnancy, cancer surveillance, surgery, and ileo-anal pouch disorders. Journal of Crohn's and Colitis. 2017 Jun 1;11(6):649-70.

4. Feuerstein JD, Cheifetz AS. Ulcerative colitis: epidemiology, diagnosis, and management. Mayo Clin Proc. 2014, 89(11):1553-63.

5. Pharmacopoeia of the People's Republic of China. National Pharmacopoeia Commission [S]. 1. Beijing: Chemical industry press, 2015:33.

6. Xu FQ, Du XB, Yao MN. Protective Effects of Aqueous Extract from Portulacaoleracea L.on Ulcerative Colitis. Progress in Modern Biomedicine, 2016, 16(32):6239-6243.

7. Prideaux L, Kamm M A, De Cruz P. Inflammatory bowel disease in Asia: A systematic review. Gastroenterol Hepatol, 2012, 27(8): 1266-1280.

8. Høivik M L, Moum B, Solberg IC. Work disability in inflammatory bowel disease patients 10 years after disease onset: results from the IBSEN Study. Gut, 2013, 62(3): 368-375.

9. [9] Gajendran M, Loganathan P, Jimenez G, Catinella AP, $\mathrm{Ng} \mathrm{N}$, Umapathy C, Ziade N, Hashash JG. A comprehensive review and update on ulcerative colitis. Dis Mon. 2019,
65(12): 10085 .

10. Rabbenou W, Ullman TA. Risk of Colon Cancer and Recommended Surveillance Strategies in Patients with Ulcerative Colitis. Gastroenterol Clin North Am. 2020, 49(4):791-807.

11. Iranshahy M, Javadi B, Iranshahi M, Jahanbakhsh SP, Mahyari S, Hassani FV, Karimi G. A review of traditional uses, phytochemistry and pharmacology of Portulaca oleracea L. J Ethnopharmacol. 2017, 205:158-172.

12. Rahimi VB, Ajam F, Rakhshandeh H, Askari VR. A Pharmacological Review on Portulaca oleracea L.: Focusing on Anti-Inflammatory, Anti-Oxidant, Immuno-Modulatory and Antitumor Activities. J Pharmacopuncture. 2019, 22(1):7-1.

13. Zhou YX, Xin HL, Rahman K, Wang SJ, Peng C, Zhang H. Portulaca oleracea L.: a review of phytochemistry and pharmacological effects. Biomed Res Int. 2015, 2015:925631.

14. Li Y, Hu Y, Shi S, Jiang L. Evaluation of antioxidant and immuno-enhancing activities of Purslane polysaccharides in gastric cancer rats. International journal of biological macromolecules. 2014 Jul 1;68:113-6.

15. Miao L, Tao H, Peng Y, Wang S, Zhong Z, ElSeedi H, Dragan S, Zengin G, San Cheang W, Wang Y, Xiao J. The anti-inflammatory potential of Portulaca oleracea L.(purslane) extract by partial suppression on NF- $\kappa \mathrm{B}$ and MAPK activation. Food chemistry. 2019 Aug 30;290:23945. 